\title{
GENUS SPECTRA FOR SPLIT METACYCLIC GROUPS
}

\author{
A. WEAVER* \\ 800 Riverside Drive, Apt. 6B, New York, N.Y. 10032, USA \\ e-mail: aweaver436@aol.com
}

(Received 28 September, 1999)

\begin{abstract}
An integer $n \geq 2$ is said to be a genus of a finite group $G$ if there is a compact Riemann surface of genus $n$ on which $G$ acts as a group of automorphisms. In this paper, formulae are given for the minimum genus, minimum stable genus and the gap sequence, i.e., the (finite) set of non-genera, for a split metacyclic group of order $p q$, where $p$ and $q$ are primes. This information completely determines the genus spectrum for such groups.
\end{abstract}

1991 Mathematics Subject Classification. Primary: 14J50; Secondary: 20B25.

1. Introduction. The genus spectrum of a finite group $G$ is the set of all integers $n \geq 2$ such that $G$ acts effectively as a group of biholomorphic homeomorphisms (automorphisms) of some compact Riemann surface of genus $n$. It was shown by Kulkarni [7] that, given a finite group $G$, there exists a positive integer $N_{G}$, determined by the Sylow subgroup structure of $G$, such that the following statements hold.

- The genus spectrum contains only integers of the form $1+m N_{G}$, where $m$ is a positive integer.

- Except for finitely many $m$, all integers of this form belong to the genus spectrum.

If $1+m N_{G}$ belongs to the genus spectrum, $m$ is called a reduced genus of $G$. There is a minimum reduced genus, $\mu_{0}$, and a minimum stable reduced genus; that is, a smallest integer $\sigma_{0}$ such that all $m \geq \sigma_{0}$ are reduced genera for $G$. The integers in the interval $\left[\mu_{0}, \sigma_{0}\right]$ that are not reduced genera are called, collectively, the reduced gap sequence of $G$. The genus spectrum is completely determined by $\mu_{0}$ and the reduced gap sequence ( $\sigma_{0}$ being one more than the last element in the reduced gap sequence).

The minimum genus has been determined for several families of groups, namely, cyclic [6], non-cyclic abelian [10], alternating and symmetric [2], metacyclic [4] (with an error), $P S L_{2}(p)$ [5], $S L_{2}\left(F_{q}\right)$ [12], and most of the sporadic simple groups [3]. (In some of these cases, the elements of the group are allowed to reverse the orientation of the surface.) The complete genus spectrum is known only for cyclic groups of order a power of a prime [8], elementary abelian $p$-groups, $p$-groups of cyclic $p$ deficiency $\leq 2$, and certain other $p$-groups [11]. In this paper, we determine the genus spectrum of a semi-direct product of a cyclic group of order $p$ with a cyclic group of order $q$, where $p$ and $q$ are primes. In the special case in which $q=2 p+1$, these groups arise as the maximal solvable subgroups of $\operatorname{PSL}_{2}(q)$.

* Research supported by a grant from the City University of New York PSC-CUNY Research Award Program. 
For the groups under consideration in this paper, and indeed all finite groups whose Sylow subgroups are all cyclic, $N_{G}=1$. It is convenient, in these cases, to avoid the adjective "reduced", since a reduced genus is just an actual genus, less 1. Accordingly, in this paper, $\mu$ denotes the (actual) minimum genus, $\sigma$ the (actual) minimum stable genus; the gap sequence is the set of genera in the interval $[\mu, \sigma]$ in which the group does not act.

2. Fuchsian Groups. In discussing Riemann surface automorphism groups it is convenient to use the language of Fuchsian groups. We sketch the necessary background. For further details, the reader is referred to [9]. ments

A group $\Gamma$ is said to have signature $\left(h ; r_{1}, \ldots r_{n}\right)$ if it is generated by $2 h+n$ ele-

$$
a_{1}, b_{1}, \ldots, a_{h}, b_{h}, x_{1}, \ldots, x_{n}
$$

where $x_{i}$ has order $r_{i}>1(i=1, \ldots, n)$ and

$$
\prod_{i=1}^{h}\left[a_{i}, b_{i}\right] \prod_{j=1}^{n} x_{j}=1 .
$$

The $r_{i}$ are called, collectively, the periods of $\Gamma$. If the Euler characteristic

$$
\chi(\Gamma)=2 h-2+\sum_{i=1}^{n}\left(1-\frac{1}{r_{i}}\right)
$$

is positive, $\Gamma$ is called Fuchsian. In this case, $\Gamma$ can be realized as a group of isometries of the hyperbolic plane, acting properly discontinuously with fundamental domain having area equal to $2 \pi \chi(\Gamma)$. A Fuchsian group with no periods is called a surface group, since it is isomorphic to the fundamental group of a compact surface of genus $h$. A surface group of genus $h$ (i.e., having signature $(h ;-)$ ), is denoted by $K_{h}$.

A finite group $G$ acts as a group of automorphisms of a compact Riemann surface of genus $g \geq 2$ if and only if $G$ is the image of a Fuchsian group $\Gamma$ under a surface-kernel epimorphism (epimorphism whose kernel is a surface group) with kernel $K_{g}$. We say that $\Gamma$ covers the action of $G$. The signature of $\Gamma$ is also called the data of the $G$ action. The data and the order $|G|$ of $G$ are related by the Riemann Hurwitz relation

$$
\chi\left(K_{g}\right)=|G| \cdot \chi(\Gamma)
$$

3. Split metacyclic groups. Let $p$ and $q$ be primes. The split metacyclic group $D_{p q}$ of order $p q$ has presentation

$$
D_{p q}=\left\langle a, b \mid a^{q}=b^{p}=1, b a b^{-1}=a^{r}\right\rangle,
$$

where $r$ is any solution (other than 1) to the congruence 


$$
r^{p} \equiv \bmod q
$$

Such solutions exist if and only if $p$ divides $q-1$. There are exactly $p-1$ non-trivial solutions; different non-trivial solutions give rise to isomorphic groups [1]. When $p=2, r=-1$ is a non-trivial solution for all $q$. In this case, the group is dihedral of order $2 q$.

The commutator subgroup of $D_{p q}$ is the cyclic group of order $q$ generated by the element $a$. All other proper subgroups constitute a single conjugate class of cyclic groups of order $p$, generated by the elements $a^{j} b a^{-j}, j=1, \ldots, q-1$, respectively. Thus the Sylow subgroups are cyclic. Every element of $D_{p q}$ can be written in the form $b^{j} a^{i}$, for some $0 \leq j<p, 0 \leq i<q$; an element has order $q$ if and only if $j=0$; otherwise, it has order $p$.

If there exists a surface-kernel epimorphism from a Fuchsian group $\Gamma$ to $D_{p q}$, the periods of $\Gamma$, if any, are $p$ and $q$. Let $\Gamma$ have signature $\left(h ; p^{[t]}, q^{[s]}\right)$, where the bracketed superscripts give the number of occurrences of the periods $p$ and $q$. Thus $\Gamma$ has generators

$$
a_{1}, b_{1}, \ldots, a_{h}, b_{h}, x_{1}, \ldots, x_{t}, y_{1}, \ldots, y_{s}
$$

and relations

$$
x_{i}^{p}=1, \quad y_{j}^{q}=1, \quad \prod_{k=1}^{h}\left[a_{k}, b_{k}\right] \prod_{i=1}^{t} x_{i} \prod_{j=1}^{s} y_{j}=1 .
$$

The existence of a surface-kernel epimorphism from $\Gamma$ requires that $D_{p q}$ be generated by $h$ pairs of arbitrary elements (the images of the $a_{k}, b_{k}$ under the epimorphism), $t$ elements of order $p$ (images of the $x_{i}$ ), and $s$ elements of order $q$ (images of the $y_{j}$ ).

THeORem 3.1. Let $\Gamma$ be a Fuchsian group with signature $\left(h ; p^{[t]}, q^{[s]}\right)$. There exists a surface-kernel epimorphism from $\Gamma$ to $D_{p q}$ if and only if the following conditions are satisfied.

1. If $\Gamma$ is a surface group (i.e., $t+s=0$ ), then $h \geq 2$.

2. If $\Gamma$ is not a surface group and $t=0$, then $h \geq 1$.

3. If $t \neq 0, t \geq 2$.

4. If $p=2$, $t$ is even.

Proof. We shall prove that the conditions are necessary; sufficiency is proved by explicit construction of surface-kernel epimorphisms, for which the reader is referred to [4].

The first condition is necessary simply because a surface group of genus 1 is not Fuchsian $\left(\chi\left(K_{1}\right)=0\right)$. For the necessity of the second condition, suppose if possible that $h=0, t=0$, and $s \geq 1$. The images of the $s$ elements $y_{j}$ lie in the commutator subgroup $\left(D_{p q}\right)^{\prime}=\langle a\rangle$, and hence cannot generate the group. Thus the covering is not an epimorphism, contrary to assumption. For the necessity of the third condition suppose, if possible, that $t=1$. The image in $D_{p q}$ of the long relation in (2) requires that the image of $y_{1}$ has order $p$, a contradiction. For the fourth condition, if $p=2$ and $t$ is odd, $\chi(\Gamma)$ is not an integer. 
4. The case in which $p$ is odd. Throughout this section, $p$ and $q$ denote fixed odd primes, $p \mid(q-1)$;

$$
M=\frac{q-p}{2} ; \quad P=\frac{p-1}{2} .
$$

Let $n \geq 2$ be in the genus spectrum of $D_{p q}$. Then there exists a Fuchsian group $\Gamma$ with signature $\left(x ; p^{[t]}, q^{[s]}\right)$ (subject to the restrictions in Theorem 3.1) that contains the surface group $K_{n}$ as a normal subgroup of index $p q$. By the Riemann-Hurwitz relation (1)

$$
2 n-2=p q\left[2 x-2+t\left(1-\frac{1}{p}\right)+s\left(1-\frac{1}{q}\right)\right] .
$$

For $(x, y) \in \mathbb{Z} \times \mathbb{Z}$ let

$$
\operatorname{sig}(x, y)=\left\{\left(x ; p^{[y-i]}, q^{[i]}\right) \mid i=0, \ldots, y\right\}
$$

be the set of signatures having genus $x$ and a total of $y$ periods $p, q$. We shall call a pair $(x, y)$ Fuchsian if $\operatorname{sig}(x, y)$ contains at least one Fuchsian signature. For $p, q$ odd, the set of Fuchsian pairs is the set

$$
\{(x, y) \in \mathbb{Z} \times \mathbb{Z} \mid x, y \geq 0 \text { and }(x, y) \neq(0,0),(0,1),(0,2),(1,0)\} .
$$

Putting $t=y-i, s=i$ in equation (3) and solving for $n$, one obtains

$$
n=g(x, y)+i M,
$$

where

$$
g(x, y)=1+p q(x-1)+y q P .
$$

In (4) we keep only those values of $i$ in the range $0,1, \ldots, y$ that produce a Fuchsian

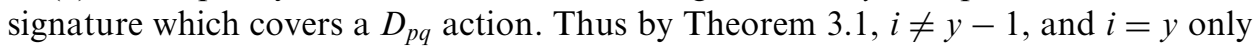
if $x \neq 0$. The portion of the $D_{p q}$ spectrum so obtained is denoted by $\operatorname{spec}(x, y)$. Precisely, if $(x, y)$ is not Fuchsian, $\operatorname{spec}(x, y)=\emptyset$; otherwise

$$
\begin{array}{r}
\operatorname{spec}(x, y)=\{g(x, y)+i M \mid \\
i=0 \text { if } y=0, \\
i=1 \text { if } y=1, \\
i=0,1, \ldots, y-2 \text { if } y \geq 2 \text { and } x=0, \\
i=0,1, \ldots, y-2, \widehat{y-1}, y \text { if } y \geq 2 \text { and } x>0\} .
\end{array}
$$

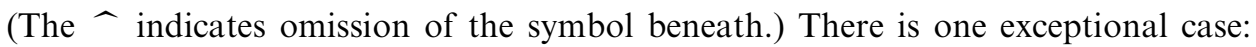
$\operatorname{spec}(0,3)$ when $p=3$. In this instance, we must exclude $i=0$, since the signature $(0 ; 3,3,3)$ is not Fuchsian.

Lemma 4.1. If $(x, y)$ is a Fuchsian pair, then $g(x, y) \geq g(0,3)$, where $g$ is the function defined in (5). 
Proof. The statement is easily verified in the following three situations, which cover all Fuchsian pairs: (i) $x \geq 2, y \geq 0$; (ii) $x=1, y \geq 1$; (iii) $x=0, y \geq 3$.

It follows that the minimum genus for $D_{p q}$ is the first element in $\operatorname{spec}(0,3)$. Taking into account the special definition of $\operatorname{spec}(0,3)$ when $p=3$, we obtain the following result.

Corollary 4.2. The minimum genus of a $D_{p q}$ action ( $p, q$ odd) is

$$
\mu= \begin{cases}1+\frac{q-3}{2} & \text { if } p=3 \\ 1+q\left(\frac{p-3}{2}\right) & \text { if } p>3 .\end{cases}
$$

(In [4] the minimum genus was stated incorrectly, except in the case $p=3$.)

The function $g: \mathbb{Z} \times \mathbb{Z} \rightarrow \mathbb{Z}$ defined by (5) induces an equivalence relation $(\sim)$ on $\mathbb{Z} \times \mathbb{Z}$, defined by

$$
(x, y) \sim\left(x^{\prime}, y^{\prime}\right) \Longleftrightarrow g(x, y)=g\left(x^{\prime}, y^{\prime}\right) .
$$

Lemma 4.3. Let $\overline{(x, y)}$ denote the class of $(x, y)$ in $\mathbb{Z} \times \mathbb{Z} / \sim$.

1. $\overline{(x, y)}=\{(x-j P, y+j p) \mid j \in \mathbb{Z}\}$.

2. There is a unique representative $(\bar{x}, \bar{y})$ of the class $\overline{(x, y)}$ with the property that $0 \leq \bar{x} \leq P-1$.

3. If $(x, y)$ is a Fuchsian pair, then so is $(\bar{x}, \bar{y})$.

Proof. Suppose that $(x+a, y+b)$ is a representative of $\overline{(x, y)}$, where $a, b \in \mathbb{Z}$. Then $0=g(x+a, y+b)-g(x, y)=p q a+b q P$ and so $\frac{a}{b}=-\frac{P}{p}$. Clearly $(a, b)$ is a solution if and only if $a=-j P, b=j p$, for some $j \in \mathbb{Z}$. In particular if $j_{0}$ is the largest integer not exceeding $x / P$, then

$$
(\bar{x}, \bar{y})=\left(x-j_{0} P, y+j_{0} p\right)
$$

is the unique equivalent pair with $0 \leq \bar{x} \leq P-1$. Finally, if $(x, y)$ is Fuchsian, $y \geq 0$ and $j_{0} \geq 0$. We assume that $j_{0} \geq 1$; otherwise there is nothing to prove. Then $\bar{y}=y+j_{0} p \geq j_{0} p \geq p \geq 3$. Thus $\bar{x}, \bar{y} \geq 0$, and $(\bar{x}, \bar{y}) \neq(0,0),(0,1),(0,2),(1,0)$; i.e., $(\bar{x}, \bar{y})$ is Fuchsian.

$g$ induces a total order on $\mathbb{Z} \times \mathbb{Z}$ defined by

$$
(x, y) \leq\left(x_{1}, y_{1}\right) \Longleftrightarrow g(x, y) \leq g\left(x_{1}, y_{1}\right) .
$$

Lemma 4.4. In the total order induced by $g,(x+1, y-2)$ is an immediate successor of $(x, y)$. Moreover, $g(x+1, y-2)-g(x, y)=q$.

Proof. Suppose that $(x+a, y+b)$ is an immediate successor of $(x, y)$ in the ordering induced by $g$. Then the difference $g(x+a, y+b)-g(x, y)=p q a+b q P$ is equal to a minimal positive integer $m$. Since $p q a+b q P$ is divisible by $q$, so is $m$. Let $m$ be as small as possible; i.e., $m=q$. Then $p a+b P=1$, which has $a=1, b=-2$ as a solution. 
Consider the sequence of pairs

$$
(i, 3-2 i) \quad(i=0,1,2, \ldots)
$$

which starts at $(0,3)$ and whose $j$ th term is an immediate successor of the $(j-1)$ th term (Lemma 4.4). Using (8) we obtain the (termwise equivalent) sequence

$$
\left(a_{i}, b_{i}\right)=(\bar{i}, \overline{3-2 i}), \quad(i=0,1,2, \ldots),
$$

in which every pair equivalent to a Fuchsian pair is actually Fuchsian. To obtain a formula that is easier to work with, let $t_{i}$ and $s_{i}$ be the quotient and remainder, respectively, upon division of $i$ by $P$. Then

$$
\begin{aligned}
\left(a_{i}, b_{i}\right) & =\left(s_{i}, 3+t_{i}-2 s_{i}\right), \quad i=0,1,2, \ldots, \\
i & =t_{i} P+s_{i}, \quad 0 \leq s_{i} \leq P-1 .
\end{aligned}
$$

The significance of this sequence is described in the next result.

Lemma 4.5. $n \in D_{p q}$ spectrum $\Longleftrightarrow n \in \operatorname{spec}\left(a_{i}, b_{i}\right)$, for some $i \geq 0$.

Proof. Only the forward implication needs proof. Suppose that $n \in D_{p q}$ spectrum. Then $n \in \operatorname{spec}(x, y)$ for some Fuchsian pair $(x, y)$, and so $n=g(x, y)+i M$ for some $i \leq y$. We first show that $n \in \operatorname{spec}(\bar{x}, \bar{y})$ as well. Since $i<\bar{y}$, by (8), this will follow as long as $i \neq \bar{y}-1$. If $i=\bar{y}-1$, then $\bar{y}-y=i+1-y \leq 1$, which is only possible if $\bar{y}=y$. But then $i=y-1$ implies that $n \notin \operatorname{spec}(x, y)$, a contradiction. It remains to show that $(\bar{x}, \bar{y})=\left(a_{i}, b_{i}\right)$ for some $i \geq 0$. Define $s_{i}=\bar{x}$ and $t_{i}=-3+\bar{y}+2 \bar{x}$. Note that $t_{i} \geq 0$, since $(\bar{x}, \bar{y})$ is Fuchsian. Then $(\bar{x}, \bar{y})=\left(a_{i}, b_{i}\right)$, where $i=t_{i} P+s_{i} \geq 0$.

The elements of $\operatorname{spec}\left(a_{i}, b_{i}\right)$ all belong to the residue class of $g\left(a_{i}, b_{i}\right) \bmod M$. Consider the sequence $\{g(i)\}_{i=0}^{\infty}$, where $g(i)=g\left(a_{i}, b_{i}\right)$. From (9),

$$
g(i)=1+q(P-1+i) .
$$

Because the sequence increases by increments of $q$, the first $M$ elements determine a complete set of residues $\bmod M$, and, for each $0 \leq k \leq M-1,\{g(k+j M)\}_{j=0}^{\infty}$ is the subsequence in the residue class of $g(k)$. Thus the portion of the genus spectrum in the class of $g(k)$ is $\cup_{j=0}^{\infty} \operatorname{spec}\left(a_{k+j M}, b_{k+j M}\right)$, and the complete spectrum is the union of these sets for $k=0,1, M-1$.

Let $\operatorname{gap}(i)$ be the complement of $\operatorname{spec}\left(a_{i}, b_{i}\right)$ in $\{g(i)+j M \mid j=0,1, \ldots, q-1\}$. $\operatorname{gap}(i)$ is the set of integers in the residue class of $g(i)$ lying between the last element of $\operatorname{spec}\left(a_{i}, b_{i}\right)$ and the first element of $\operatorname{spec}\left(a_{i+M}, b_{i+M}\right)$, when the latter two sets are non-empty. (A more explicit definition of $g a p(i)$ is given at (12).) Since

$$
g(i+M)-g(i)=q M,
$$

(6) implies that $g a p(i)$ is non-empty whenever $b_{i} \leq q$.

Lemma 4.6 The portion of the $D_{p q}$ gap sequence in the residue class of $g(k)$, $0 \leq k \leq M-1$, is 


$$
X_{k} \cup\left\{\bigcup_{j=0}^{D} \operatorname{gap}(k+j M)\right\},
$$

where $D$ is a finite integer less than $C=P(q+p-5)-1$, and $X_{k}$ is the set $\{x \in \mathbb{Z} \mid \mu<x<g(k), x \equiv g(k) \bmod M\}$. ( $\mu$ is the minimum genus (7).)

Proof. It is clear from the definition that the elements of $\operatorname{gap}(k+j M), j \geq 0$, belong to the gap sequence and to the residue class of $g(k) \bmod M$. To prove that $X_{k}$ is a subset of the gap sequence, suppose to the contrary that $n \in D_{p q}$ spectrum and also $n \in X_{k}$ for some $0 \leq k \leq P-1$. Then $n \in \operatorname{spec}(x, y)$ for a pair $(x, y)$ equivalent to $\left(a_{k-j M}, b_{k-j M}\right)$, where $j>0$. But $g(x, y)=g\left(a_{k-j M}, b_{k-j M}\right)<g(0,3)$ and so, by Lemma 4.1, $(x, y)$ is not Fuchsian and $\operatorname{spec}(x, y)=\emptyset$, a contradiction.

Conversely, if $n$ belongs to the gap sequence, and $n \equiv g(k) \bmod M$ for some $0 \leq k \leq M-1$, then either

1. $n \geq g(k)$ and $n \in\{g(k+l M)+j M \mid j=0,1, \ldots, q-1\}$ for some $l \geq 0$, which implies that $n \in \operatorname{gap}(k+l M)$; or

2. $\mu<n<g(k)$, which implies that $n \in X_{k}$.

We now show that $\operatorname{gap}(i)$ is empty for all sufficiently large $i$. By (6), gap $(i)$ is empty if $b_{i}>q$. Let $i$ be a positive integer such that $b_{i+1}, b_{i+2}, b_{i+3} \ldots>q$. Then, from (9), $3+t_{i+1}-2 s_{i+1} \geq 3+t_{i+1}-2(P-1)>q$ and

$$
\begin{aligned}
t_{i+1} & >q+p-6 \\
i & >P(q+p-5)-1 .
\end{aligned}
$$

Thus $C=P(q+p-5)-1$ is the smallest positive integer such that, for all $i>C, \operatorname{gap}(i)$ is empty.

It follows that the complete gap sequence is the union of the sets (10) for $k=0,1, \ldots, M-1$. To state this result, we use more explicit definitions of $\operatorname{gap}(i)$ and $X_{i}$.

Let $N_{i}$ be the greatest integer not exceeding $(g(i)-\mu) / M$.

$$
\begin{aligned}
& X_{i}=\emptyset \text { if } i>M-1 \text { or } N_{i} \leq 0 ; \text { otherwise } \\
& X_{i}=\left\{g(i)-\left(N_{i}-j\right) M \mid j=0,1, \ldots, N_{i}-1\right\} .
\end{aligned}
$$

As before, $t_{i}$ and $s_{i}$ are the quotient and remainder, respectively, of $i$ upon division by $P$,

$$
\begin{aligned}
& i=t_{i} P+s_{i}, \quad 0 \leq s_{i} \leq P-1, \\
& g(i)=1+q(P-1+i), \\
& b_{i}=3+t_{i}-2 s_{i} . \\
& \operatorname{gap}(i)=\emptyset \text { if } b_{i}>q ;
\end{aligned}
$$

otherwise

$$
g a p(i)=\{g(i)+j M \mid j \in J\}
$$

where 


$$
J= \begin{cases}\{0, \ldots, q-1\} & \text { if } \quad b_{i}<0 ; \\ \{1, \ldots, q-1\} & \text { if } b_{i}=0 \\ \left\{b_{i}-1, b_{i}, b_{i}+1, \ldots, q-1\right\} & \text { if } 0<b_{i} \leq q \text { and } s_{i}=0 \\ \left\{b_{i}-1, \widehat{b}_{i}, b_{i}+1, \ldots, q-1\right\} & \text { if } \quad 0<b_{i} \leq q \text { and } s_{i}>0\end{cases}
$$

(The $\widehat{-}$ indicates omission of the symbol beneath.)

Theorem 4.7. The $D_{p q}$ gap sequence ( $p, q$ odd) is

$$
\bigcup_{i=0}^{C}\left\{X_{i} \cup \operatorname{gap}(i)\right\},
$$

where $C=P(q+p-5)-1 ; X_{i}$ and gap(i) are defined at (11) and (12), respectively.

COROllary 4.8. The minimum stable genus of a $D_{p q}$ action ( $p, q$ odd) is

$$
\sigma=2+p q(P-2)+q^{2} P+(q-1) M .
$$

Proof. From Lemma 4.6, $\operatorname{gap}(C)$ is the last non-empty set in the sequence $\{g a p(i)\}$. Since $C=P(q+p-6)+P-1$, by (9), $b_{C}=q$ and $g a p(C)$ is the singleton $\{g(C)+(q-1) M\}$ containing the largest integer in the gap sequence; the minimum stable genus is one more than this integer. Hence (13) is established.

5. The case $p=2$. We state the results for $D_{2 q}$ and only sketch the proof. The method is analogous to the case in which $p$ is odd.

For $i=0,1,2, \ldots$, let $t_{i}$ and $s_{i}$ be the quotient and remainder, respectively, upon division of $i$ by 2 . Let $h(i)=1+t_{i} q+s_{i}(q-1)$, and $N_{i}$ the greatest integer not exceeding $(h(i)-(q-1)) /(q-2)$. Define

$$
X_{i}=\emptyset \text { if } N_{i} \leq 0
$$

otherwise

$$
\begin{gathered}
X_{i}=\left\{h(i)-\left(N_{i}-j\right)(q-2) \mid j=0,1, \ldots, N_{i}-1\right\} \\
g a p(i)=\emptyset \text { if } i>q-5
\end{gathered}
$$

otherwise

$$
\operatorname{gap}(i)=\left\{h(i)+j(q-2) \mid j=t_{i}+2, t_{i}+3, \ldots, \frac{q-1}{2}-s_{i}\right\} .
$$

TheOREM 5.1. For $D_{2 q}$, where $q$ is an odd prime, we have

1. the minimum genus is $\mu=q-1$;

2. the minimum stable genus is

$$
\sigma= \begin{cases}2 & \text { if } q=3 \\ (q-1)(q-3) & \text { otherwise }\end{cases}
$$


3. the gap sequence is

$$
\bigcup_{i=0}^{q-3}\left\{X_{i} \cup \operatorname{gap}(i)\right\},
$$

where $X_{i}$ and $g a p(i)$ are defined at (14) and (15), respectively.

Proof. (Sketch) When $p=2$ the function (5) can take half-integral values, but still induces an equivalence relation on pairs. It is easy to show that every pair $(x, y)$ is equivalent to a pair in which $x=0$, and so we need only consider the sets $\operatorname{spec}(0, y), y \geq 3$. Theorem 3.1 implies that $\left(0 ; 2^{[y-l]}, q^{l}\right)$ is a signature covering a $D_{2 q}$ action only if $l \equiv y(\bmod 2)$. This condition guarantees the elements of $\operatorname{spec}(0, y)$ are integers. Denoting $y$ by $y=4+i, i=0,1, \ldots$, and letting $t_{i}, s_{i}$ be the quotient and remainder, respectively, of $i$ upon division by 2 , we obtain the more convenient

$$
\operatorname{spec}(i)=\left\{h(i)+j(q-2) \mid j=0,1, \ldots, t_{i}+1\right\},
$$

where $h(i)=1+q t_{i}+s_{i}(q-1)$. We do not allow $y=3$ since $(0 ; 2,2, q)$ is not a Fuchsian signature. Similarly $(0 ; 2,2,2,2)$ is not Fuchsian, so that in $\operatorname{spec}(0), i \neq 0$. It follows that the minimum genus of $D_{2 q}$ is the first (and only) admissible element in $\operatorname{spec}(0)$, which is $q-1$.

Evidently the elements of spec(i) all belong to the residue class of $h(i) \bmod (q-2)$. It is not hard to see that for $k=0,1, \ldots, q-3,\{h(k)+j(q-2)\}_{j=0}^{\infty}$ is the subsequence of $\{h(i)\}$ in the residue class $\bmod (q-2)$ of $h(k)$. Since $h(i+q-2)-h(i)=\left(\frac{q+1}{2}-s_{i}\right)(q-2)$, we define $\operatorname{gap}(i)$ to be the complement of $\operatorname{spec}(i)$ in the set $\left\{h(i)+j(q-2) \mid j=0,1, \ldots, \frac{q+1}{2}-s_{i}-1\right\}$, which is (15).

The last nonempty sets in the sequence $\{\operatorname{gap}(i)\}$ occur when $t_{i}+2=\frac{q-1}{2}-s_{i}$, which is the case when $i=q-5-s_{i}$; i.e., when $i=q-6, q-5$. Thus the last element in the gap sequence is the single integer in $\operatorname{gap}(q-5)$; one more than this integer is the minimum stable genus $(q-1)(q-3)$. If $q=3$, the gap sequence is empty and $\sigma=\mu=2$.

The sets $X_{k}, k=0,1, \ldots, q-3$, complete the gap sequence with integers $\mu<n<h(k)$ in the residue class of $h(k) \bmod (q-2)$.

In a future paper, the techniques used here will be adapted to cyclic groups of order $p q$.

\section{REFERENCES}

1. W. Burnside, Theory of groups of finite order (Dover Publications, 1955).

2. M. D. E. Conder, The symmetric genus of alternating and symmetric groups, $J$. Combin. Theory Ser. B 39 (1985), 179-186.

3. M. D. E. Conder, R. A. Wilson and A. J. Woldar, The symmetric genus of sporadic groups, Proc. Amer. Math. Soc. 116 (1992), 653-663.

4. S. K. Dutta, K. Patra and B. P. Chetiya, ZS-metacyclic groups of automorphisms of compact Riemann surfaces, Indian J. Pure Appl. Math. 28 (1997), 63-74.

5. H. Glover and D. Sjerve, Representing $P S L_{2}(p)$ on a Riemann surface of least genus, Enseign Math. 31 (1985), 305-325. 
6. W. J. Harvey, Cyclic groups of automorphisms of compact Riemann surfaces, Quart. J. Math. Oxford Ser. 217 (1966), 86-97.

7. R. S. Kulkarni, Symmetries of surfaces, Topology 26 (1987), 195-203.

8. R. S. Kulkarni and C. Maclachlan, Cyclic p-groups of symmetries of surfaces, Glasgow Math J. 33 (1991), 213-221.

9. A. M. Macbeath, Discontinuous groups and birational transformations, in Proceedings of the Summer School in Geometry and Topology (Queens College, Dundee, 1961), 59-75.

10. C. Maclachlan, Abelian groups of automorphisms of compact Riemann surfaces, Proc. London Math. Soc. (3) 15 (1965), 699-712.

11. C. Maclachlan and Y. Talu, p-groups of symmetries of surfaces, Michigan Math. J. 45 (1998), 315-332.

12. S. N. Voon, The genus of $S L_{2}\left(F_{q}\right)$, Michigan Math. J. 40 (1993), 527-544. 